\title{
IP-RIGIDITY AND EIGENVALUE GROUPS
}

\author{
JON. AARONSON, MARYAM HOSSEINI \& MARIUSZ LEMAŃCZYK
}

\begin{abstract}
We examine the class of increasing sequences of natural numbers which are IP-rigidity sequences for some weakly mixing probability preserving transformation. This property is closely related to the uncountability of the eigenvalue group of a corresponding non-singular transformation. We give examples, including a super-lacunary sequence which is not IP-rigid.
\end{abstract}

\section{$\S 0$ InTroduction}

Let $(X, \mathcal{B}, m)$ be a standard, continuous, probability space (that is, $(X, \mathcal{B})$ is a Polish space equipped with its Borel sets and a non-atomic $m \in \mathcal{P}(X)$ (the collection of probability measures on $(X, \mathcal{B})$ ).

We'll denote by MPT $=\operatorname{MPT}(X, \mathcal{B}, m)$ the collection of invertible, probability preserving transformations of $(X, \mathcal{B}, m)$. This is a Polish space when equipped with the coarse topology with basic neighborhoods of form

$$
\begin{aligned}
U\left(T_{0}, f_{1}, \ldots, f_{N} ; \epsilon\right) & := \\
& \left\{T \in \mathrm{MPT}:\left\|f_{j} \circ T^{s}-f_{j} \circ T_{0}^{s}\right\|_{L^{2}(m)}<\epsilon \forall 1 \leq j \leq N, s= \pm 1\right\}
\end{aligned}
$$

where $T_{0} \in \operatorname{MPT}(X, \mathcal{B}, m)$ and $f_{1}, \ldots, f_{N} \in L^{2}(m)$.

Equipped with the coarse topology, $\operatorname{MPT}(X, \mathcal{B}, m)$ is a topological group under composition. It is embedded into the Polish, topological group $\mathcal{U}\left(L^{2}(m)\right)$ of unitary operators on $L^{2}(m)$ equipped with the strong operator topology by the Koopman representation $U_{T} f:=f \circ$ $T\left(T \in \operatorname{MPT}(X, \mathcal{B}, m), f \in L^{2}(m)\right)$. Accounts of the spectral theory of unitary operators can be found in $[\mathrm{KT}]$ and [N2].

Date: May 24th 2012.

2010 Mathematics Subject Classification. 37A05, (37A30, 37A40).

Key words and phrases. Probability preserving transformation, spectral type, rigidity, IP-convergence, Dirichlet measure, eigenvalue group.

Aaronson and Hosseini would like to thank Nicolaus Copernicus University, and Aaronson the University of Surrey, for hospitality provided when this paper was done. Aaronson's research was partially supported by Israel Science Foundation grant No. 1114/08. 


\section{Recurrence and rigidity.}

A sequence $q \in \mathbb{N}^{\mathbb{N}}(\uparrow):=\left\{q \in \mathbb{N}^{\mathbb{N}}: q_{n}<q_{n+1} \forall n \geq 1\right\}$ is called a sequence of recurrence for $T \in \operatorname{MPT}(X, \mathcal{B}, m)$ if $\lim _{\sup _{n \rightarrow \infty}} \mu\left(A \cap T^{-q_{n}} A\right)>$ 0 for each $A \in \mathcal{B}$ of positive measure.

Rigidity is a stronger version of recurrence.

An sequence $q \in \mathbb{N}^{\mathbb{N}}(\uparrow)$ is called a rigidity sequence for $T \in \operatorname{MPT}(X, \mathcal{B}, m)$ if $\mu\left(T^{q_{n}} A \triangle A\right) \rightarrow 0$ for each $A \in \mathcal{B}$; equivalently

$$
T^{q_{n}} \underset{n \rightarrow \infty}{\stackrel{\mathrm{MPT}}{\longrightarrow}} \mathrm{Id} .
$$

Using spectral theory one sees that $(X)$ is equivalent to the restricted spectral type $\sigma_{T}$ of $T$ (i.e. $\left.U_{T}\right|_{L^{2}(m)_{0}}$ ) having the Dirichlet property along $q$, that is

$$
\chi_{q_{n}} \underset{n \rightarrow \infty}{\stackrel{L^{2}\left(\mathbb{T}, \sigma_{T}\right)}{\longrightarrow}} 1
$$

where $\chi_{k}(t):=e^{2 \pi i k t}$.

Rigidity sequences for non-trivial transformations must be sparse. In particular, unless $T \in$ MPT is purely periodic any rigidity sequence for $T$ has at most finite intersection with each of its translates whence has zero Banach density.

Additional properties of rigidity sequences are studied in [BJLR] \& [EG] including the rigidity properties of lacunary and super-lacunary sequences, a sequence $q \in \mathbb{N}^{\mathbb{N}}(\uparrow)$ being called lacunary if $\frac{q_{n+1}}{q_{n}} \geq \lambda>$ $1 \forall n \geq 1$ and super-lacunary if $\frac{q_{n+1}}{q_{n}} \underset{n \rightarrow \infty}{\longrightarrow} \infty$.

Rigid factors, mild mixing and IP sets. Let $T \in \operatorname{MPT}(X, \mathcal{B}, m)$ and let $q \in \mathbb{N}^{\mathbb{N}}(\uparrow)$. It is well known that the collection of sets

$$
\mathcal{R}(q):=\left\{A \in \mathcal{B}: m\left(A \Delta T^{q_{n}} A\right) \underset{n \rightarrow \infty}{\longrightarrow} 0\right\}
$$

is a $T$-invariant, $\sigma$-algebra. It corresponds to the maximal factor of $T$ which is rigid along $q$. The transformation $T \in \operatorname{MPT}(X, \mathcal{B}, m)$ is called mildly mixing if it has no non-trivial, rigid factor along any $q \in \mathbb{N}^{\mathbb{N}}(\uparrow)$ (as in $[\mathrm{FW}]$ ).

Since the spectral type $\sigma_{S}$ of a factor $S$ of $T$ is absolutely continuous with respect to $\sigma_{T}$, it is evident that $T$ has some non-trivial rigid factor if and only if $\exists$ a Dirichlet measure $\mu \ll \sigma_{T}$, (that is, one satisfying ( $\ddagger$ ) along some $q \in \mathbb{N}^{\mathbb{N}}(\uparrow)$ ).

An IP-set is a collection of "finite sum sets" of form

$$
\mathrm{FS}(q):=\{q(F): F \in \mathcal{F}\}
$$


where $q \in \mathbb{N}^{\mathbb{N}}(\uparrow)$ and for

$$
F \in \mathcal{F}:=\{\text { finite, nonempty subsets of } \mathbb{N}\}, q(F):=\sum_{j \in F} q_{j} .
$$

This notion appears in combinatorics, ultrafilter theory, topological dynamics (see $[\mathrm{Fu}]$ and $[\mathrm{HS}]$ ) and also in ergodic theory.

As shown in $[\mathrm{Fu}], T \in \operatorname{MPT}(X, \mathcal{B}, m)$ is mildly mixing if and only if $\exists K \subset \mathbb{N}$ which intersects with every finite sum set so that

$$
m\left(A \cap T^{-n} B\right) \underset{n \rightarrow \infty, n \in K}{\longrightarrow} m(A) m(B) \forall A, B \in \mathcal{B} ;
$$

equivalently (see [HMP1]),

$T$ is not mildly mixing if and only if $\exists q \in \mathbb{N}^{\mathbb{N}}(\uparrow)$ so that

$$
\inf _{n \in \mathrm{FS}(\mathrm{q})}|\widehat{\mu}(n)|>0 .
$$

The considerations involved give rise to the notion of

\section{IP convergence.}

Let $q \in \mathbb{N}^{\mathbb{N}}(\uparrow)$. We'll say that a sequence $a: \mathbb{N} \rightarrow Z$ (a metric space) converges IP to $L \in Z$ along $\mathrm{FS}(q)$

$$
\begin{gathered}
\text { written } a(n) \underset{n \rightarrow \infty}{\stackrel{\mathrm{FS}(q)}{\longrightarrow}} L, \text { in } Z \text { if } \\
a(q(F)) \underset{F \in \mathcal{F}, \min F \rightarrow \infty}{\longrightarrow} L .
\end{gathered}
$$

This paper is about

\section{IP-rigidity.}

We'll say that $b \in \mathbb{N}^{\mathbb{N}}(\uparrow)$ is

an $I P$-rigidity sequence for $T$ and that $T$ is $I P$-rigid along $b$ if

$$
T^{n} \underset{n \rightarrow \infty}{\stackrel{\mathrm{FS}(b)}{\longrightarrow}} \text { Id in MPT. }
$$

Let

IPRWM $:=\left\{b \in \mathbb{N}^{\mathbb{N}}(\uparrow): \exists T \in \operatorname{MPT}(X, \mathcal{B}, m)\right.$, weakly mixing \& IP-rigid along $\left.b\right\}$.

Any rigid transformation is IP-rigid on some subsequence (see $[\mathrm{Fu}]$ ). On the other hand if a transformation is IP-rigid on $q \in \mathbb{N}^{\mathbb{N}}(\uparrow)$, then it is rigid along much thicker subsequences (see $§ 5$ ).

Similarly to $(\dot{\delta})$, for $q \in \mathbb{N}^{\mathbb{N}}(\uparrow)$, the collection

$$
\mathcal{R}_{\mathrm{IP}}(q):=\left\{A \in \mathcal{B}: m\left(A \Delta T^{n} A\right) \underset{n \rightarrow \infty}{\stackrel{\mathrm{FS}(q)}{\longrightarrow}} 0\right\}
$$

is a $T$-invariant, $\sigma$-algebra. It corresponds to the maximal factor of $T$ which is IP-rigid along $q$. As above, $T$ has a non-trivial factor, IP-rigid along $q$ if and only if $\lim _{N \rightarrow \infty} \inf _{F \in \mathcal{F}, \min F \geq N}\left|\widehat{\sigma_{T}}(q(F))\right|>0$. 
The existence of IP-Dirichlet measures along $b \in \mathbb{N}^{\mathbb{N}}(\uparrow)$ is related to the groups

$$
\begin{aligned}
& G_{p}(b):=\left\{t \in \mathbb{T}: \sum_{n=1}^{\infty}\left\|b_{n} t\right\|^{p}<\infty\right\}(0<p<\infty) \& \\
& G_{\infty}(b):=\left\{t \in \mathbb{T}:\left\|b_{n} t\right\| \underset{n \rightarrow \infty}{\longrightarrow} 0\right\}
\end{aligned}
$$

where for $x \in \mathbb{R},\|x\|:=\min _{n \in \mathbb{Z}}|x-n|$.

These groups are discussed in [AN] and [HMP2].

\section{Results.}

Proposition 1 Suppose that $b \in \mathbb{N}^{\mathbb{N}}(\uparrow)$, then

$$
\left|G_{1}(b)\right|>\aleph_{0} \Longrightarrow b \in \text { IPRWM. }
$$

Proposition 1 (which is folklore) can be proved using Propositions 1.1 and 1.2 (below).

Theorem 2 If $b \in \mathbb{N}^{\mathbb{N}}(\uparrow)$, then

$$
b \in \mathrm{IPRWM} \Longrightarrow\left|G_{2}(b)\right|>\aleph_{\mathbf{0}} .
$$

Theorem 2 also provides an answer to a question in [BJLR]:

$(-) \quad$ if $b \in$ IPRWM, then some irrational rotation is rigid along $b$ because if $\left|G_{2}(b)\right|>\aleph_{0}$ then $\exists \alpha \in G_{2}(b) \backslash \mathbb{Q}$. It follows that rotation of $\mathbb{T}$ by $\alpha$ is rigid along $b$. $\nabla$

The converse of theorem 2 holds for arithmetic sequences and Erdos-Taylor sequences for different reasons.

A sequence $q \in \mathbb{N}^{\mathbb{N}}(\uparrow)$ is called arithmetic if it is either

- multiplicative in the sense that $q_{n} \mid q_{n+1} \forall n \geq 1$; or it is the

- principal denominator sequence of some $\alpha \in \mathbb{T} \backslash \mathbb{Q}$, being defined by $q_{0}=1, q_{1}=a_{1}, q_{n+1}:=a_{n+1} q_{n}+q_{n-1}$ where $\alpha=\left[0 ; a_{1}, a_{2}, \ldots\right]$ is the continued fraction expansion of $\alpha$.

Proposition 3 Let $b \in \mathbb{N}^{\mathbb{N}}(\uparrow)$, be arithmetic. The following statements are equivalent.
(a) $\varlimsup_{n \rightarrow \infty} \frac{b_{n+1}}{b_{n}}=\infty$.
(b) $\left|G_{1}(b)\right|>\aleph_{0}$.
(c) $b \in$ IPRWM. (d) $\left|G_{2}(b)\right|>\aleph_{\mathbf{0}}$. 
The Erdos-Taylor sequence associated to $\left(a_{1}, a_{2}, \ldots\right) \in \mathbb{N}^{\mathbb{N}}$ is $b=$ $\left(b_{1}, b_{2}, \ldots\right) \in \mathbb{N}^{\mathbb{N}}(\uparrow)$ defined by

$$
b_{1}:=1 . \quad b_{n+1}:=a_{n} b_{n}+1 .
$$

Erdos-Taylor sequences were introduced in [ET] and are considered to be "extremely non-arithmetic".

Proposition 4 If $b \in \mathbb{N}^{\mathbb{N}}(\uparrow)$, is an Erdos-Taylor sequence, then
(i) $\sum_{n \geq 1}\left(\frac{b_{n}}{b_{n+1}}\right)^{2}<\infty \Longleftrightarrow$
(ii) $b \in$ IPRWM $\Longleftrightarrow$
(iii) $\left|G_{2}(b)\right|>\aleph_{0}$.

We'll see that there are super-lacunary Erdos-Taylor sequences $b \& q \epsilon$ $\mathbb{N}^{\mathbb{N}}(\uparrow)$ satisfying $\left|G_{2}(b)\right|>\aleph_{0} \& G_{1}(b)=\{0\}$ and $G_{2}(q)=\{0\}$.

\section{Eigenvalue Groups and theorem 2.}

Groups of form $G_{2}$ appear as eigenvalue groups (see [AN]). Eigenvalue groups and rigidity are related as follows:

An ergodic probability preserving transformation $S$ is not mildly mixing (i.e. has a rigid factor) if and only if there is a conservative, ergodic non-singular transformation $T$ so that $S \times T$ is not ergodic (see $[\mathrm{FW}]$ ). By the ergodic multiplier theorem of M. Keane (see e.g. $\$ 2.7$ of $[\mathrm{A}])$, this situation is characterized by $\sigma_{S}(e(T))>0$ where $\sigma_{S}$ is the restricted spectral type of $S$ and $e(T)$ is the group of eigenvalues of $T$.

We prove Theorem 2 in $\S 4$ by considering a dyadic cocycle (see below) associated to $b \in \mathbb{N}^{\mathbb{N}}(\uparrow)$ over the dyadic adding machine. The eigenvalue group of the Mackey range (as in p. 76-77 in [Z]) of this cocycle is $G_{2}(b)$. In case $b$ is a growth sequence as in [A2], that is $b(n)>\sum_{1 \leq k<n} b(k)$, then the Mackey range preserves a $\sigma$-finite measure and is isomorphic to the appropriate dyadic tower over the dyadic adding machine (defined in [A2]).

\section{Organization of the paper.}

In $\S 1$ we establish the basic results on Dirichlet sets and measures and begin to consider membership of IPRWM.

In $\S 2$ we consider the class of arithmetic sequences, and prove Proposition 3 .

In $\S 3$ we prove proposition 4 for Erdos-Taylor sequences and give our main examples.

The proofs of propositions $3 \& 4$ both use Theorem 2 which is established in $\S 4$. In $\S 5$ we make some quantitative remarks on the growth of rigid sequences for transformations IP-rigid along some (particular) $b \in \mathbb{N}^{\mathbb{N}}(\uparrow)$. 


\section{$\S 1$ DiRichlet SETS AND MEASURES}

Dirichlet sets. A Dirichlet set is a subset $\Gamma \subset \mathbb{T}$ of form

$$
\Gamma(b)=\left\{t \in \mathbb{T}: \chi_{b_{n}}(t) \underset{n \rightarrow \infty}{\longrightarrow} 1\right\}
$$

where $b \in \mathbb{N}^{\mathbb{N}}(\uparrow) \& \quad \chi_{n}(t):=e^{2 \pi i n t}$.

An $I P$-Dirichlet set is a subset $\Gamma \subset \mathbb{T}$ of form

$$
\Gamma(\mathrm{FS}(b))=\left\{t \in \mathbb{T}: \chi_{n}(t) \underset{n \rightarrow \infty}{\stackrel{\operatorname{FS}(b)}{\longrightarrow}} 1\right\}
$$

where $b \in \mathbb{N}^{\mathbb{N}}(\uparrow)$. Here, we have

Proposition 1.1 For $b \in \mathbb{N}^{\mathbb{N}}(\uparrow)$,

$$
\Gamma(\mathrm{FS}(b))=G_{1}(b) .
$$

\section{Proof sketch of $\subseteq$}

It suffices to show that for $t \in \mathbb{R}$,

$$
\|n t\| \underset{n \rightarrow \infty}{\stackrel{\mathrm{FS}(b)}{\longrightarrow}} 0 \Rightarrow \sum_{n \geq 1}\left\|b_{n} t\right\|<\infty .
$$

For $x \in \mathbb{R}$, let $\lfloor x\rceil$ be the nearest integer to $x$ (if there are two, take the lesser one), and let

$$
\langle x\rangle:=x-\lfloor x\rceil, \text { then }|\langle x\rangle|=\|x\| \leq \frac{1}{2} .
$$

Fix $t \in \mathbb{R}$ so that $\|n t\| \underset{n \rightarrow \infty}{\stackrel{\text { FS }(b)}{\longrightarrow}} 0$, let $K>0$ be so that

$$
\|b(F) t\|<\frac{1}{16} \quad \forall F \in \mathcal{F}, \min F \geq K .
$$

If $F, G \subset[K, \infty) \cap \mathbb{N}$ are disjoint finite sets, then

$$
\|b(F) t\|,\|b(G) t\|,\|b(F \bullet G) t\|<\frac{1}{16} .
$$

Since $\langle b(F \cup G) t\rangle-\langle b(F) t\rangle-\langle b(G) t\rangle \in \mathbb{Z}$, this forces $\langle b(F \cup G) t\rangle=\langle b(F) t\rangle+\langle b(G) t\rangle$.

It follows that

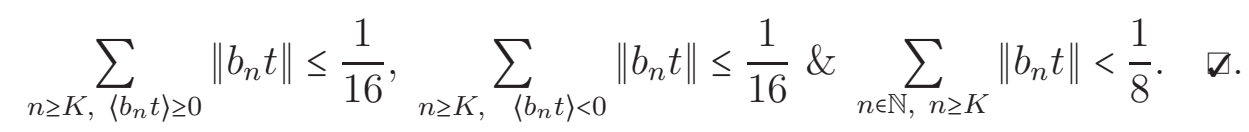


Dirichlet measures. A probability measure $\mu \in \mathcal{P}(\mathbb{T})$ is called

- a Dirichlet measure if

$$
\left\|\chi_{b_{n}}-1\right\|_{L^{2}(\mu)} \underset{n \rightarrow \infty}{\longrightarrow} 0
$$

for some $b \in \mathbb{N}^{\mathbb{N}}(\uparrow)$ in which case $\mu$ is called Dirichlet along $b$ and

- an IP Dirichlet measure if

$$
\left\|\chi_{n}-1\right\|_{L^{2}(\mu)} \underset{n \rightarrow \infty}{\stackrel{\mathrm{FS}(b)}{\longrightarrow}} 0
$$

for some $b \in \mathbb{N}^{\mathbb{N}}(\uparrow)$ in which case $\mu$ is called IP-Dirichlet along $b$.

Evidently:

$\chi_{n_{k}} \underset{k \rightarrow \infty}{\stackrel{L^{2}(\mu)}{\longrightarrow}} 1$ if and only if $\widehat{\mu}\left(n_{k}\right) \underset{k \rightarrow \infty}{\longrightarrow} \mu(\mathbb{T})$,

if $\mu$ is IP-Dirichlet along $b$, then so is any $\nu \ll \mu$,

if $\mu$ is IP-Dirichlet, then $\exists b \in \mathbb{N}^{\mathbb{N}}(\uparrow)$ so that $\sum_{n \geq 1}\left\|\chi_{b_{n}}-1\right\|_{L^{2}(\mu)}<\infty$, whence $\mu\left(G_{1}(b)\right)=1$ and $\mu$ is IP-rigid along $b$.

By Proposition 1.1, a totally atomic measure $\mu \in \mathcal{P}(\mathbb{T})$ is IP-Dirichlet along $b$ if and only if $\mu\left(G_{1}(b)\right)=1$. Examples in $\S 4$ (below) show that this is false for continuous measures $\mu \in \mathcal{P}(\mathbb{T})$.

\section{Proposition 1.2}

$$
\text { IPRWM }=\left\{b \in \mathbb{N}^{\mathbb{N}}(\uparrow): \exists \mu \in \mathcal{P}(\mathbb{T}) \text { continuous \& IP-Dirichlet along } b\right\} .
$$

Proof of $\subseteq$ Suppose that $b \in \operatorname{IPRWM}$ and that $(X, \mathcal{B}, m, T)$ is a weakly mixing, probability preserving transformation so that $T^{n} \underset{n \rightarrow \infty}{\stackrel{\operatorname{FS}(b)}{\longrightarrow}} \operatorname{Id}$. Fix $f \in L^{2}(m), \int_{X} f d m=0, \int_{X}|f|^{2} d m=1$. The spectral measure of $f$ : $\mu \in \mathcal{P}(\mathbb{T})$ is continuous and IP-Dirichlet along $b$.

Proof of $\supseteq \quad$ Suppose that $\mu_{0} \in \mathcal{P}(\mathbb{T})$ is continuous and IP-Dirichlet along $b$. Let $\mu$ be the symmetrization of $\mu_{0}$ (also continuous and IPDirichlet along $b)$ and let $(X, \mathcal{B}, m, T)$ be the shift of the Gaussian process with spectral measure $\mu$. The spectral type of $(X, \mathcal{B}, m, T)$ is $\sigma_{T}=\sum_{n \geq 0} \mu^{n *}$ where $\mu^{n *}$ denotes the $n$-fold convolution of $\mu$ with itself (see e.g. [CFS]). Each $\mu^{K *}$ is continuous (whence $T$ is weakly mixing) and IP-Dirichlet along $b$ (since $\left.\widehat{\mu^{K *}}(n)=\widehat{\mu}(n)^{K} \underset{n \rightarrow \infty}{\stackrel{\text { FS }(b)}{\longrightarrow}} 1\right)$. Every $\nu \ll \sigma_{T}$ is also IP-Dirichlet along $b$ and $T$ is IP-rigid along $b$. Thus $b \in$ IPRWM.

$\square$ 
It follows from proposition 1.2 that if $b \in \mathbb{N}^{\mathbb{N}}(\uparrow)$ is an IP rigidity sequence for some $T \in$ MPT not of discrete spectrum (i.e. $\sigma_{T}$ is not totally atomic) then $b \in$ IPRWM.

We complete this section with a "mixed" multiplicative-finite sum condition for membership in IPRWM.

Proposition 1.3 Suppose that $b \in \mathbb{N}^{\mathbb{N}}(\uparrow)$ and that $\exists S \subset \mathbb{N}$ infinite, so that $\sum_{n \in S} \frac{b_{n}}{b_{n+1}}<\infty$, and $b_{n} \mid b_{n+1}$ for $n \notin S$, then $b \in$ IPRWM.

If $\mathbb{N} \backslash S$ is finite, then $\left|G_{1}(b)\right|>\aleph_{0}$ by Theorem 5 in [ET], whence $b \in$ IPRWM by proposition 1 .

Proof Assume (without loss of generality) that $b_{1}=1$. We construct a weakly mixing $T \in \operatorname{MPT}(X, \mathcal{B}, m)$ which is IP-rigid along $b$ by cutting and stacking as in Ch. 7 of [N1].

To this end, we construct a nested sequence of Rokhlin towers $\left(\tau_{n}\right)_{n \geq 1}$ of intervals where $\tau_{n}$ has height $b_{n}$.

Let $\tau_{1}$ be $[0,1]$. To construct $\tau_{n+1}$ from $\tau_{n}$ :

- if $n \notin S \& b_{n+1}=a_{n} b_{n}, a_{n} \in \mathbb{N}, a_{n} \geq 2$ then we cut $\tau_{n}$ into $a_{n}$ columns and stack.

- If $n \in S \& b_{n+1}=a_{n} b_{n}+r_{n}, a_{n}, r_{n} \in \mathbb{N}, 1 \leq r_{n}<b_{n}$, we cut $\tau_{n}$ into $a_{n}$ columns and put one spacer interval above the $\left\lfloor\frac{a_{n}}{2}\right\rfloor$ 'th column from the left and $r_{n}-1$ spacer intervals above the last column in the right and then we stack.

The tower $\tau_{n}$ is a stack of $b_{n}$ intervals of length $\prod_{j=1}^{n-1} \frac{1}{a_{j}}$ called levels of $\tau_{n}$.

It follows from $\S 7.31$ in $[\mathrm{N} 1]$ that the transformation $T$ constructed preserves a finite measure $m$. A standard argument as in the proof of Proposition 3.10 of [BJLR] shows that $T$ is weakly mixing.

Next, we show that if $A$ is a union of levels in some $\tau_{K}$, then

$$
\sum_{n=1}^{\infty} m\left(A \Delta T^{b_{n}} A\right)<\infty
$$

To see this, we note first that $A$ is also a union of levels in every $\tau_{n}(n \geq K)$. Fix $n \geq K$ and write

$$
S \cap[K, \infty)=\left\{s_{1}<s_{2}<s_{3}<\ldots\right\} .
$$

Since $\frac{1}{a_{s_{\ell}}+1} \leq \frac{b_{s_{\ell}}}{b_{s_{\ell}+1}} \leq \frac{1}{a_{s_{\ell}}}$ our assumptions imply $\sum_{\ell=1}^{\infty} \frac{1}{a_{s_{\ell}}}<\infty$.

To estimate $m\left(A \Delta T^{-b_{n}} A\right)$ for $n \in\left(s_{\ell-1}, s_{\ell}\right]$ we consider the appearance of the tower $\tau_{n}$ as " $\tau_{n}$-stalks" inside $\tau_{s_{\ell}+1}$. 
A $\tau_{n}$-stalk in $\tau_{s_{\ell}+1}$ is a union $\mathfrak{s}=\bigcup_{k=0}^{b_{n}-1} T^{k} B$ of levels of $\tau_{s_{\ell}+1}$ where $B$ is contained in the base of $\tau_{n}$.

Let $\mathfrak{s} \in \mathcal{B}$ be a $\tau_{n}$-stalk in $\tau_{s_{\ell}+1}$, then

$$
m(\mathfrak{s} \cap A)=\prod_{j=n}^{s_{\ell}} \frac{1}{a_{j}} \cdot m(A) .
$$

By construction, $\tau_{s_{\ell}+1}$ consists entirely of $\tau_{n}$-stalks and spacer stalks added in $\tau_{s_{\ell}+1}$. Thus, all points of $A$ except those contained in the two $\tau_{n}$-stalks preceding the spacer stalks added in $\tau_{s_{\ell}+1}$, return to $A$ at time $b_{n}$, so

$$
m\left(A \Delta T^{-b_{n}} A\right)=2 m\left(A \backslash T^{-b_{n}} A\right)=4 \prod_{j=n}^{s_{\ell}} \frac{1}{a_{j}} \cdot m(A) \leq \frac{4}{2^{s_{\ell}-n}} \cdot \frac{1}{a_{s_{\ell}}} \cdot m(A) .
$$

Thus, writing $s_{0}:=K-1$, we have

$$
\begin{aligned}
\sum_{n=K}^{\infty} m\left(A \Delta T^{-b_{n}} A\right) & =\sum_{\ell=1}^{\infty} \sum_{n \in\left(s_{\ell-1}, s_{\ell}\right]} m\left(T^{b_{n}}(A) \Delta A\right) \\
& \leq \sum_{\ell=1}^{\infty} \sum_{n \in\left(s_{\ell-1}, s_{\ell}\right]} \frac{4}{2^{s_{\ell}-n}} \cdot \frac{1}{a_{s_{\ell}}} \cdot m(A) \\
& \leq 4 m(A) \sum_{\ell=1}^{\infty} \frac{1}{a_{s_{\ell}}} \\
& <\infty .
\end{aligned}
$$

It follows from this that for $A$ a union of levels in some tower $\tau_{n}$ and $F=\left\{n_{1}<n_{2}<\cdots<n_{k}\right\} \in \mathcal{F}$,

$$
\begin{aligned}
m\left(A \Delta T^{b(F)} A\right) & =m\left(A \Delta T^{\sum_{j=1}^{k} b_{n_{j}}} A\right) \\
& \leq m\left(A \Delta T^{b_{1}} A\right)+m\left(T^{b_{1}} A \Delta T^{\sum_{j=1}^{k} b_{n_{j}}} A\right) \\
& =m\left(A \Delta T^{b_{1}} A\right)+m\left(A \Delta T^{\left.\sum_{j=2}^{k} b_{n_{j}} A\right)}\right. \\
& \leq \\
& \vdots \\
& \leq \sum_{j=1}^{k} m\left(A \Delta T^{b_{j}} A\right) \\
& \leq \sum_{n=\min F}^{\infty} m\left(T^{b_{n}}(A) \Delta A\right) \\
& \underset{F \in \mathcal{F}, \min F \rightarrow \infty}{\longrightarrow} 0 .
\end{aligned}
$$


The collection of measurable sets $\mathcal{C}$ satisfying this last convergence is a $\sigma$-algebra and

$$
\mathcal{C} \supset \sigma\left(\bigcup_{n \geq 1}\left\{\text { unions of levels in } \tau_{n}\right\}\right)=\mathcal{B} .
$$

Thus $T$ is IP-rigid along $b$.

Remark 1.4. The proof of Proposition 1.3 establishes the following proposition:

Suppose that $b \in \mathbb{N}^{\mathbb{N}}(\uparrow), p>0$ and that $\exists S \subset \mathbb{N}$ infinite, so that $\sum_{n \in S}\left(\frac{b_{n}}{b_{n+1}}\right)^{p}<\infty$, and $b_{n} \mid b_{n+1}$ for $n \notin S$, then $\exists T \in \operatorname{MPT}(X, \mathcal{B}, m)$ weakly mixing and a dense collection $\mathcal{A} \subset \mathcal{B}$ so that

$$
\sum_{n \geq 1} m\left(A \Delta T^{b_{n}} A\right)^{p}<\infty \quad \forall A \in \mathcal{A} .
$$

As above, $\psi(1) \Longrightarrow$ IP-rigidity along $b$ whence $\sigma_{T}\left(G_{2}(b)^{c}\right)=0$ by Theorem 2. Using the spectral theorem, one sees that $\Psi\left(\frac{1}{2}\right) \Longrightarrow$ $\sigma_{T}\left(G_{1}(b)^{c}\right)=0$.

\section{$\S 2$ Arithmetic sequences}

In this section, we prove proposition 3. The implications $(\mathrm{b}) \Longrightarrow(\mathrm{c})$ $\Longrightarrow(\mathrm{d}) \Longrightarrow(\mathrm{a})$ follow from proposition 1 , theorem 2 and theorem 16 in $[\mathrm{E}]$ (respectively). None of these uses arithmeticity.

We turn to the remaining implication $(\mathrm{a}) \Longrightarrow(\mathrm{b})$.

\section{Lemma 2.1}

If $b \in \mathbb{N}^{\mathbb{N}}(\uparrow)$ is multiplicative and $\sup _{n \geq 1} \frac{b_{n+1}}{b_{n}}=\infty$, then $\left|G_{1}(b)\right|>\aleph_{\mathbf{0}}$.

C.f. theorem 3 in $[\mathrm{ET}]$.

Proof Suppose that $b_{n+1}=a_{n+1} b_{n}$, where $a_{n} \geq 2 \forall n \geq 1$.

Since $\sup _{n \geq 1} a_{n}=\infty, \exists$ a subsequence $\left(n_{k}\right)_{k \geq 1}$ such that $a_{n_{k+1}} / a_{n_{k}} \geq$ $3 \forall k \geq 1$. Define $t: \Omega:=\{0,1\}^{\mathbb{N}} \rightarrow[0,1]$ by

$$
t(\omega):=\sum_{k=1}^{\infty} \frac{\omega_{k}}{b_{n_{k}}}
$$

Since

$$
\sum_{k=L+1}^{\infty} \frac{\omega_{k}}{b_{n_{k}}} \leq \frac{1}{b_{n_{L}}} \sum_{j=1}^{\infty} \frac{1}{a_{n_{L}+1} a_{n_{L}+2} \cdots a_{n_{j}}} \leq \frac{1}{b_{n_{L}}} \sum_{j=1}^{\infty} \frac{1}{3^{j}}<\frac{1}{b_{n_{L}}}
$$

we have that $t: \Omega:=\{0,1\}^{\mathbb{N}} \rightarrow[0,1]$ is strictly increasing (with respect to lexicographic order on $\Omega$ ), whence injective and $|t(\Omega)|>\aleph_{\mathbf{0}}$.

It suffices to show that $t(\Omega) \subset G_{1}(b)$. 
To see this, fix $\omega \in \Omega$. For $N \geq 1$, we have that

$$
b_{N} t(\omega)=\sum_{k=1}^{\infty} \frac{b_{N} \omega_{k}}{b_{n_{k}}}=\sum_{k \geq 1, n_{k} \geq N} \frac{\omega_{k}}{a_{N+1} a_{N+2} \cdots a_{n_{k}}} \bmod 1,
$$

whence

$$
\left\|b_{N} t(\omega)\right\|=\sum_{k \geq 1, n_{k} \geq N} \frac{\omega_{k}}{a_{N+1} a_{N+2} \cdots a_{n_{k}}} \leq \sum_{k \geq 1, n_{k} \geq N} \frac{1}{a_{N+1} a_{N+2} \cdots a_{n_{k}}}=: \Delta_{N} .
$$

For $n_{K-1}<N \leq n_{K}$,

$$
\Delta_{N}=\sum_{k \geq K} \frac{1}{a_{N+1} a_{N+2} \cdots a_{n_{k}}} \leq \sum_{k \geq K} \frac{1}{a_{N+1} a_{N+2} \cdots a_{n_{K}} \cdot a_{n_{k}}} \leq \frac{1}{2^{n_{K}-N} a_{n_{K}}} .
$$

Thus

$$
\begin{aligned}
\sum_{N=n_{1}}^{\infty}\left\|b_{N} t(\omega)\right\| & \leq \sum_{N=n_{1}}^{\infty} \Delta_{N}=\sum_{k=2}^{\infty} \sum_{N=n_{k-1}+1}^{n_{k}} \sum_{\nu=k}^{\infty} \frac{1}{a_{N+1} a_{N+2} \cdots a_{n_{\nu}}} \\
& \leq \sum_{k=2}^{\infty} \sum_{N=n_{k-1}+1}^{n_{k}} \frac{1}{2^{n_{k}-N} a_{n_{k}}} \leq \sum_{k=2}^{\infty} \frac{2}{a_{n_{k}}} \leq 4
\end{aligned}
$$

and $t(\omega) \in G_{1}(b)$. $\quad \nabla$

\section{Lemma 2.2}

Let $q=q(\alpha) \in \mathbb{N}^{\mathbb{N}}(\uparrow)$ be the principal denominator sequence of $\alpha \in$ $(0,1) \backslash \mathbb{Q}$.

If $\sup _{n \geq 1} \frac{q_{n+1}}{q_{n}}=\infty$, then $\left|G_{1}(q)\right|>\aleph_{\mathbf{0}}$.

Proof As shown in [IN],

for any $t \in[0,1]$ there is a unique sequence $\left(\omega_{n}\right)_{n \geq 1} \in \prod_{n \geq 1}\left\{0,1, \cdots, a_{n}\right\}$ such that

- $\omega_{k} \leq a_{k}, \omega_{k}=a_{k} \Rightarrow \omega_{k+1}=0$ and

- $t=\sum_{n=1}^{\infty} \omega_{n}\left\langle q_{n} \alpha\right\rangle \bmod 1$.

Since $\sup _{n \geq 1} a_{n}=\sup _{n \geq 1} \frac{q_{n+1}}{q_{n}}=\infty$, we can choose a sub-sequence $a_{n_{k}}$ such that $\sum_{k \geq 1} 1 / a_{n_{k}}<\infty$ and define

$$
t: \Omega=\{0,1\}^{\mathbb{N}} \rightarrow \mathbb{T} \text { by } t(\omega):=\sum_{k=1}^{\infty} \omega_{k}\left\langle q_{n_{k}-1} \alpha\right\rangle \bmod 1
$$

By the above, $t: \Omega \rightarrow \mathbb{T}$ is injective. It follows that $|t(\Omega)|>\aleph_{\mathbf{0}}$ and it suffices to show that $t(\Omega) \subset G_{1}(q)$.

We claim that $\sup _{\omega \in \Omega} \sum_{n=1}^{\infty}\left\|q_{n} t(\omega)\right\|<\infty$. 
Fix $K \geq 1$ and consider $n_{K}-1 \leq N \leq n_{K+1}-1$. Then

$$
\begin{aligned}
\left\|q_{N} t(\omega)\right\| & \leq \sum_{k=1}^{\infty}\left\|\omega_{n_{k}-1} q_{N} q_{n_{k}-1} \alpha\right\| \\
& \leq \sum_{k=1}^{\infty}\left\|q_{N} q_{n_{k}-1} \alpha\right\| \\
& \leq \sum_{k=1}^{K} \frac{q_{n_{k}-1}}{q_{N+1}}+\sum_{k=K+1}^{\infty} \frac{q_{N}}{q_{n_{k}}} .
\end{aligned}
$$

Using the fact that $\frac{q_{j+n}}{q_{j}} \geq \sqrt{2}^{n-2} \forall j, n \geq 1$ we have for some absolute constant $C$,

$$
\frac{q_{n_{k}-1}}{q_{N+1}}=\frac{q_{n_{k}-1}}{q_{n_{K}-1}} \cdot \frac{q_{n_{K}-1}}{q_{n_{K}}} \cdot \frac{q_{n_{K}}}{q_{N+1}} \leq \frac{C}{\sqrt{2}^{n_{K}-n_{k}}} \cdot \frac{1}{a_{n_{K}}} \cdot \frac{C}{\sqrt{2}^{N-n_{K}}} \quad \text { for } k \leq K
$$

and

$$
\frac{q_{N}}{q_{n_{k}}}=\frac{q_{N}}{q_{n_{K+1}-1}} \cdot \frac{q_{n_{K+1}-1}}{q_{n_{K+1}}} \cdot \frac{q_{n_{K+1}}}{q_{n_{k}}} \leq \frac{C}{\sqrt{2}^{n_{K+1}-N}} \cdot \frac{1}{a_{n_{K+1}}} \cdot \frac{C}{\sqrt{2}^{n_{k}-n_{K+1}}} \quad \text { for } k>K .
$$

Therefore,

$$
\begin{aligned}
\sum_{N=n_{K}-1}^{n_{K+1}-2}\left\|q_{N} t(\omega)\right\| & \leq \sum_{N=n_{K}-1}^{n_{K+1}-2}\left(\sum_{k=1}^{K} \frac{q_{n_{k}-1}}{q_{N+1}}+\sum_{k=K+1}^{\infty} \frac{q_{N}}{q_{n_{k}}}\right) \\
& \leq C^{2} \sum_{N=n_{K}-1}^{n_{K+1}-2}\left(\sum_{k=1}^{K} \frac{1}{a_{n_{K}} \sqrt{2}^{N-n_{k}}}+\sum_{k=K+1}^{\infty} \frac{1}{a_{n_{K+1}} \sqrt{2}^{n_{k}-N}}\right) \\
& \leq C^{3} \sum_{N=n_{K}-1}^{n_{K+1}-2}\left(\frac{1}{a_{n_{K}} \sqrt{2}^{N-n_{K}}}+\frac{1}{a_{n_{K+1}} \sqrt{2}^{n_{K+1}-N}}\right) \\
& \leq C^{4}\left(\frac{1}{a_{n_{K}}}+\frac{1}{a_{n_{K+1}}}\right)
\end{aligned}
$$

and

$$
\sum_{N=1}^{\infty}\left\|q_{N} t(\omega)\right\| \leq C^{4} \sum_{K=1}^{\infty}\left(\frac{1}{a_{n_{K}}}+\frac{1}{a_{n_{K+1}}}\right)<\infty . \quad \nabla
$$

Hence $q \in$ IPRWM. $\quad \nabla$ The proof of Proposition 3 is now complete. $\nabla$

\section{$\S 3$ Super-LACUnARY SEQUENCES}

Suppose that $b=\left(b_{1}, b_{2}, \ldots\right) \in \mathbb{N}^{\mathbb{N}}(\uparrow)$ is super-lacunary, i.e. $\frac{b_{n+1}}{b_{n}} \underset{n \rightarrow \infty}{\longrightarrow}$ $\infty$. 
As in theorem 17 in [E], we fix $N \geq 1$ with $\frac{b_{n+1}}{b_{n}}>10 \forall n \geq N$ and let

$$
E:=\bigcap_{n \geq N} E_{n}
$$

where

Now

$$
E_{n}:=\left\{t \in[0,1]:\left\|b_{n} t\right\| \leq \frac{4 b_{n}}{b_{n+1}}\right\}
$$

where

$$
E_{n} \supseteq \bigcup_{k=1}^{b_{n}-1} I_{k, n}
$$

$$
I_{k, n}:=\left[\frac{k}{b_{n}}-\frac{4}{b_{n+1}}, \frac{k}{b_{n}}+\frac{4}{b_{n+1}}\right] .
$$

For each $n \geq N$, the intervals $\left\{I_{k, n}: 1 \leq k<b_{n}\right\}$ are disjoint, and each interval $I_{k, n}$ contains at least five disjoint intervals of form $I_{k^{\prime}, n+1}$. It follows that $E$ contains a Cantor set and $|E|>\aleph_{0}$. Thus, (c.f of theorem 5 in $[\mathrm{ET}]$ )

Proposition 3.1 Suppose that $b \in \mathbb{N}^{\mathbb{N}}(\uparrow) \& p>0$, then

$$
\sum_{n \geq 1}\left(\frac{b_{n}}{b_{n+1}}\right)^{p}<\infty \Longrightarrow\left|G_{p}(b)\right|>\aleph_{0}
$$

Proof Let $N \geq 1 \& E$ be as above, then for $t \in E$,

$$
\sum_{n \geq 1}\left\|b_{n} t\right\|^{p} \leq N+4^{p} \sum_{n \geq N}\left(\frac{b_{n}}{b_{n+1}}\right)^{p}<\infty \quad \nabla
$$

Proposition 3.2 Suppose that $b \in \mathbb{N}^{\mathbb{N}}(\uparrow)$ and that

$$
\sum_{n \geq 1}\left(\frac{b_{n}}{b_{n+1}}\right)^{2}<\infty, \text { then } b \in \text { IPRWM. }
$$

Proof By Proposition 1.2, it suffices to construct a continuous probability in $\mathbb{T}$ which is IP-Dirichlet along $b$.

To this end, let $N \geq 1, E$ and $\left\{I_{k, n}: 1 \leq k<b_{n}\right\}$ be as above. As above, for each $n \geq N$, the intervals $\left\{I_{k, n}: 1 \leq k<b_{n}\right\}$ are disjoint, and each interval $I_{k, n}$ contains at least five disjoint intervals of form $I_{k^{\prime}, n+1}$.

Thus we may choose

$$
I_{n}(\omega)=I_{k_{n}(\omega), n} \quad\left(n \geq 1, \omega \in\{0,1\}^{n}\right)
$$

so that

$$
I_{n+1}(\omega, \epsilon) \subset I_{n}(\omega) \quad \forall n \geq 1, \omega \in\{0,1\}^{n} \& \epsilon=0,1
$$

and 
$X_{n+1}(\omega, 0)<X_{n}(\omega)<X_{n+1}(\omega, 1)$ where $X_{n}(\omega):=\frac{k_{n}(\omega)}{b_{n}}$.

Next, for $\omega \in \Omega:=\{0,1\}^{\mathbb{N}}$,

$$
X_{n}\left(\omega_{1}, \ldots, \omega_{n}\right) \underset{n \rightarrow \infty}{\longrightarrow} X(\omega) \text { where } \bigcap_{n \geq 1} I_{n}\left(\omega_{1}, \ldots, \omega_{n}\right)=\{X(\omega)\}
$$

and

$$
\begin{aligned}
b_{n} X(\omega) & =b_{n} X_{n}(\omega)+b_{n}\left(X_{n+1}(\omega)-X_{n}(\omega)\right)+b_{n}\left(X(\omega)-X_{n+1}(\omega)\right) \\
& =k_{n}(\omega)+\xi_{n}(\omega)+\theta_{n}(\omega)
\end{aligned}
$$

where

$$
\begin{gathered}
\xi_{n}(\omega):=b_{n}\left(X_{n+1}(\omega)-X_{n}(\omega)\right) \& \\
\theta_{n}(\omega):=b_{n}\left(X(\omega)-X_{n+1}(\omega)\right) .
\end{gathered}
$$

Note that $\left|\theta_{n}(\omega)\right| \leq \mathcal{E}_{n}:=\frac{4 b_{n}}{b_{n+2}}$ and that by assumption, $\sum_{n \geq 1} \mathcal{E}_{n}<\infty$.

For $n \geq 1, \omega \in\{0,1\}^{n}, \exists ! p_{n, \omega}:\{0,1\} \rightarrow(0,1)$ so that

$p_{n, \omega}(0)+p_{n, \omega}(1)=1 \& X_{n+1}(\omega, 0) p_{n, \omega}(0)+X_{n+1}(\omega, 1) p_{n, \omega}(1)=X_{n}(\omega)$.

Define $P:\{$ cylinders $\} \rightarrow(0,1)$ by

$$
P\left(\left[a_{1}, a_{2}, \ldots, a_{n}\right]\right):=\frac{1}{2} \prod_{k=1}^{n-1} p_{k,\left(a_{1}, a_{2}, \ldots, a_{k}\right)}\left(a_{k}\right) .
$$

It follows that $P$ is additive and by standard extension theory $\exists \mathbb{P} \in$ $\mathcal{P}(\Omega)$ extending $P$.

Denoting expectation with respect to $\mathbb{P}$ by $\mathbb{E}$ and writing $\omega=\left(\omega_{1}, \omega_{2}, \ldots, \omega_{n}\right) \epsilon$ $\{0,1\}^{n}$, we have

$\mathbb{E}\left(X_{n+1} \| \omega_{1}, \omega_{2}, \ldots, \omega_{n}\right)=X_{n+1}(\omega, 0) p_{n, \omega}(0)+X_{n+1}(\omega, 1) p_{n, \omega}(1)=X_{n}(\omega)$,

whence

$$
\mathbb{E}\left(\xi_{n} \| \omega_{1}, \omega_{2}, \ldots, \omega_{n}\right)=b_{n}\left(\mathbb{E}\left(X_{n+1} \| \omega_{1}, \omega_{2}, \ldots, \omega_{n}\right)-X_{n}(\omega)\right)=0
$$

and $\mathbb{E}\left(\xi_{n}\right)=0$.

For $n, k \geq 1$,

$$
\begin{gathered}
\mathbb{E}\left(\xi_{n} \xi_{n+k}\right)=b_{n} b_{n+k} \mathbb{E}\left(\mathbb{E}\left(\xi_{n} \xi_{n+k} \| \omega_{1}, \omega_{2}, \ldots, \omega_{n+k}\right)\right) \\
=b_{n} b_{n+k} \mathbb{E}\left(\left(\xi_{n}(\omega) \mathbb{E} \xi_{n+k} \| \omega_{1}, \omega_{2}, \ldots, \omega_{n+k}\right)\right)=0 \quad \& \\
\mathbb{E}\left(\xi_{n}^{2}\right)=: \Delta_{n} \leq \frac{16 b_{n}^{2}}{b_{n+1}^{2}} .
\end{gathered}
$$

By assumption $\sum_{n \geq 1} \Delta_{n}<\infty$, so $\sum_{n \in K} \xi_{n}$ converges in $L^{2}(\mathbb{P})$ for every $K \subset \mathbb{N}$ and

$$
\mathbb{E}\left(\left(\sum_{n \in K} \xi_{n}\right)^{2}\right)=\sum_{n \in K} E\left(\xi_{n}^{2}\right)=\sum_{n \in K} \Delta_{n} .
$$


The measure $\mu:=\mathbb{P} \circ X^{-1} \in \mathcal{P}(\mathbb{T})$ is continuous. We claim that it is IP-Dirichlet along $b$.

To check this, let $F \subset \mathbb{N} \cap[K, \infty)$ be finite and write $\Xi_{F}:=\sum_{n \in F} \xi_{n}$, then

$$
\begin{aligned}
\left\|\chi_{b(F)}-1\right\|_{L^{2}(\mu)} & \leq\left\|\left\langle\sum_{N \in F} b_{N} t\right\rangle\right\|_{L^{2}(\mu)} \\
& =\left\|\left\langle\sum_{N \in F}\left(\xi_{N}+\theta_{N}\right)\right\rangle\right\|_{L^{2}(\mathbb{P})} \\
& \leq\left\|\left\langle\Xi_{F}\right\rangle\right\|_{L^{2}(\mathbb{P})}+\sum_{N \in F} \mathcal{E}_{N} .
\end{aligned}
$$

Next,

$$
\begin{aligned}
\left\|\left\langle\Xi_{F}\right\rangle\right\|_{L^{2}(\mathbb{P})}^{2} & =\mathbb{E}\left(\left\langle\Xi_{F}\right\rangle^{2}\right) \\
& =\mathbb{E}\left(1_{\left[\left|\Xi_{F}\right| \leq \frac{1}{2}\right]}\left\langle\Xi_{F}\right\rangle^{2}\right)+\mathbb{E}\left(1_{\left[\left|\Xi_{F}\right|>\frac{1}{2}\right]}\left\langle\Xi_{F}\right\rangle^{2}\right) \\
& \left.\leq \mathbb{E}\left(\Xi_{F}^{2}\right)+\frac{1}{4} \mathbb{P}\left[\left|\Xi_{F}\right|>\frac{1}{2}\right]\right) \quad \because\left\langle\Xi_{F}\right\rangle^{2} \leq \frac{1}{4}, \\
& \leq 2 \mathbb{E}\left(\Xi_{F}^{2}\right) \quad \text { by Tchebychev's inequality } \\
& =2 \sum_{N \in F} \mathbb{E}\left(\xi_{N}^{2}\right) \leq 2 \sum_{N=K}^{\infty} \Delta_{N} .
\end{aligned}
$$

Thus,

$$
\begin{aligned}
\left\|\chi_{n(F)}-1\right\|_{L^{2}(\mu)} & \leq\left\|\left\langle\Xi_{F}\right\rangle\right\|_{L^{2}(\mathbb{P})}+\sum_{N=K}^{\infty} \mathcal{E}_{N} \\
& \leq \sqrt{2 \sum_{N=K}^{\infty} \Delta_{N}+\sum_{N=K}^{\infty} \mathcal{E}_{N}} \\
& \underset{K \rightarrow \infty}{\longrightarrow} 0
\end{aligned}
$$

proving that $\mu$ is IP-Dirichlet along $b . \quad \nabla$

Remark. The converses to propositions $3.1 \& 3.2$ are false. It is easy to construct $b \in \mathbb{N}^{\mathbb{N}}(\uparrow)$ multiplicative, super-lacunary so that $\sum_{n \geq 1}\left(\frac{b_{n}}{b_{n+1}}\right)^{p}=\infty \quad \forall p>0$. By proposition $3,\left|G_{1}(b)\right|>\aleph_{0} \& T \in$ IPRWM.

\section{Erdos-Taylor sequences \& proposition 4.}

We begin with a strong converse to Proposition 3.1 for Erdos-Taylor sequences:

Proposition 3.3 Suppose that $b \in \mathbb{N}^{\mathbb{N}}(\uparrow)$ is an Erdos-Taylor sequence and let $p>0$, then

$$
\sum_{n \geq 1}\left(\frac{b_{n}}{b_{n+1}}\right)^{p}=\infty \quad \Longrightarrow \quad G_{p}(b)=\{0\} .
$$


This was stated in [ET] for $p=1$ and $b$ the Erdos-Taylor sequence associated to $(2,3, \ldots)$. See also Théorème 2 in $[\mathrm{P}]$.

Proof Let $b \in \mathbb{N}^{\mathbb{N}}(\uparrow)$ be the Erdos-Taylor sequence associated to $\left(a_{1}, a_{2}, \ldots\right) \in \mathbb{N}^{\mathbb{N}}$ and let $t \in \mathbb{R} \backslash \mathbb{Z}$, then

$$
\left\|b_{n} t\right\|<\frac{\|t\|}{2 a_{n}} \Longrightarrow\left\|b_{n+1} t\right\|>\frac{\|t\|}{2} .
$$

If $p>0$ and $\sum_{n \geq 1}\left(\frac{b_{n}}{b_{n+1}}\right)^{p}=\infty$, then for $t \in \mathbb{R} \backslash \mathbb{Z}$,

- either $\left\|b_{n} t\right\| \geq \frac{\|t\|}{2 a_{n}}$ eventually and $\sum_{n \geq 1}\left\|b_{n} t\right\|^{p}=\infty$, or

- $\left\|b_{n+1} t\right\|>\frac{\|t\|}{2}$ infinitely often and $\sum_{n \geq 1}\left\|b_{n} t\right\|^{p}=\infty$.

Either way, $t \notin G_{p}(b)$.

Proof of Proposition 4 The implications (i) $\Longrightarrow$ (ii) $\Longrightarrow$ (iii) $\Longrightarrow$ (i) follow from proposition 3.2, theorem 2 and proposition 3.3 (respectively).

\section{Examples.}

- If $b \in \mathbb{N}^{\mathbb{N}}(\uparrow)$ is the Erdos-Taylor sequence associated to $(2,3, \ldots)$, then

$$
\text { (a) } b \in \text { IPRWM \& (b) } G_{1}(b)=\{0\} .
$$

- If $b \in \mathbb{N}^{\mathbb{N}}(\uparrow)$ is the Erdos-Taylor sequence associated to $\left(a_{2}, a_{3}, \ldots\right)$ where $a_{n} \rightarrow \infty$ and $\sum_{n \geq 1} \frac{1}{a_{n}^{2}}=\infty$ (e.g. $a_{n}:=\lfloor\sqrt{n}\rfloor$ ), then $b$ is superlacunary, $G_{2}(b)=\{0\}$ and $b$ is not a sequence of IP-rigidity for any probability preserving transformation other than the identity.

\section{$\S 4$ Proof of Theorem 2}

We prove Theorem 2 using dyadic cocycles over the dyadic odometer.

Let $\Omega:=\{0,1\}^{\mathbb{N}}$, and let $P \in \mathcal{P}(\Omega)$ be symmetric product measure: $P=\prod\left(\frac{1}{2}, \frac{1}{2}\right)$, and let $\tau: \Omega \rightarrow \Omega$ be the dyadic odometer defined by

$$
\tau\left(1, \ldots, 1,0, \omega_{\ell+1}, \ldots\right)=\left(0, \ldots, 0,1, \omega_{\ell+1}, \ldots\right)
$$

where $\ell=\ell(\omega):=\min \left\{n \geq 1: \omega_{n}=0\right\}$.

The dyadic cocycle $\varphi: \Omega \rightarrow \mathbb{Z}$ associated to $b \in \mathbb{N}^{\mathbb{N}}(\uparrow)$ is defined by

$$
\varphi(\omega):=b_{\ell(\omega)}-\sum_{k=1}^{\ell(\omega)-1} b_{k}
$$

and its skew product

$$
\tau_{\varphi}: \Omega \times \mathbb{Z} \rightarrow \Omega \times \mathbb{Z} \text { is defined by } \tau_{\varphi}(x, n)=(\tau(x), n+\varphi(x)) .
$$


Define $Q: \Omega \times \mathbb{Z} \rightarrow \Omega \times \mathbb{Z}$ by $Q(x, n):=(x, n+1)$ and fix $p \in \mathcal{P}(\Omega \times$ $\mathbb{Z}), p \sim P \times \#$ where $\#$ is counting measure on $\mathbb{Z}$.

There is (see [Z], pp. 76-77) an ergodic, non-singular transformation $(\mathfrak{X}, \mathcal{B}(\mathfrak{X}), \mathfrak{q}, \mathfrak{T})$ of a standard probability space and a map $\pi: \Omega \times \mathbb{Z} \rightarrow \mathfrak{X}$ so that

$$
p \circ \pi^{-1}=\mathfrak{q}, \quad \pi^{-1} \mathcal{B}(\mathfrak{X})=\left\{A \in \mathcal{B}(\Omega \times \mathbb{Z}): \tau_{\varphi} A=A\right\} \quad \& \quad \pi \circ Q=\mathfrak{T} \circ \pi .
$$

The ergodic, non-singular transformation $(\mathfrak{X}, \mathcal{B}(\mathfrak{X}), \mathfrak{q}, \mathfrak{T})$ is called the Mackey range of $(\tau, \varphi)$.

In case $b$ is a growth sequence, equivalently $\varphi: \Omega \rightarrow \mathbb{N}$, there is a $\sigma$-finite, invariant $T$-invariant measure $\mathfrak{m} \sim \mathfrak{q}$ with respect to which the Mackey range $(\mathfrak{X}, \mathcal{B}(\mathfrak{X}), \mathfrak{m}, \mathfrak{T})$ is isomorphic to the tower over $(\Omega, \mathcal{B}(\Omega), P, \tau)$ with height function $\varphi$ (aka the dyadic tower with growth sequence $b$ in $[\mathrm{A} 2])$.

The collection of eigenvalues of the Mackey range is

$$
e(\mathfrak{T}):=\left\{t \in \mathbb{T}: \exists F \in L^{\infty}(\mathfrak{q}), F \neq \equiv, F \circ \mathfrak{T}=e^{2 \pi i t} F\right\}
$$

and it follows from the definitions that

$$
e(\mathfrak{T})=\mathcal{T}(\tau, \varphi):=\left\{s \in \mathbb{T}: \exists f \in L^{\infty}(P), f \not \equiv 0, \quad f \circ \tau=e^{2 \pi i s \varphi} f\right\} .
$$

It it is shown in $\S 2$ of $[\mathrm{AN}]$ (see also Theorem 2.6.3 of [A1]) that

$$
\mathcal{T}(\tau, \varphi)=G_{2}(b) .
$$

Although formally, ( was only stated for growth sequences in [AN] and [A1], the proofs do not use this condition and apply to arbitrary $b \in \mathbb{N}^{\mathbb{N}}(\uparrow)$.

Consider the Polish group $\mathfrak{B}(\mu):=\left\{f \in L^{2}(\mu):|f| \equiv 1\right\}$ equipped with $L^{2}(\mu)$-distance.

\section{Lemma 4.1}

If the probability $\mu \in \mathcal{P}(\mathbb{T})$ is IP Dirichlet along b, then $\exists \mathcal{X}: \Omega \rightarrow$ $\mathfrak{B}(\mu)$ continuous so that

$$
\sup _{\omega \in \Omega}\left\|\chi_{b(K(\omega) \cap[1, n])}-\mathcal{X}(\omega)\right\|_{L^{2}(\mu)} \underset{n \rightarrow \infty}{\longrightarrow} 0
$$

where $K(\omega):=\left\{n \geq 1: \omega_{n}=1\right\}$.

\section{Proof}


Suppose that $\mu \in \mathcal{P}(\mathbb{T})$ is IP Dirichlet along $b$. Fix $\omega \in \Omega$. We claim that the sequence $n \mapsto \chi_{b(K(\omega) \cap[1, n])}$ is Cauchy in $L^{2}(\mu)$. To see this, let

$$
\mathcal{E}_{n}:=\sup _{F \in \mathcal{F}, \min F \geq n}\left\|\chi_{b(F)}-1\right\|_{L^{2}(\mu)}
$$

then by assumption $\mathcal{E}_{n} \underset{n \rightarrow \infty}{\longrightarrow} 0$. Evidently

$$
\left\|\chi_{b(K(\omega) \cap[1, n])}-\chi_{b(K(\omega) \cap[1, n+k])}\right\|_{L^{2}(\mu)}=\left\|\chi_{b(K(\omega) \cap[n+1, n+k])}-1\right\|_{L^{2}(\mu)} \leq \mathcal{E}_{n}
$$

whence $\exists \mathcal{X}: \Omega \rightarrow \mathfrak{B}(\mu)$ so that

$$
\chi_{b(K(\omega) \cap[1, n])} \underset{n \rightarrow \infty}{\stackrel{L^{2}(\mu)}{\longrightarrow}} \mathcal{X}(\omega) \text { uniformly in } \omega \in \Omega .
$$

For $\omega \in \Omega$,

$\left\|\chi_{b(K(\omega) \cap[1, n])}-\mathcal{X}(\omega)\right\|_{L^{2}(\mu)} \underset{k \rightarrow \infty}{\longleftarrow}\left\|\chi_{b(K(\omega) \cap[1, n])}-\chi_{b(K(\omega) \cap[1, n+k])}\right\|_{L^{2}(\mu)} \leq 2 \mathcal{E}_{n}$

proving $(\$)$. Clearly, for each $n \geq 1, \omega \mapsto \chi_{b(K(\omega) \cap[1, n])}$ is continuous $(\Omega \rightarrow \mathfrak{B}(\mu))$ and so continuity of $\mathcal{X}: \Omega \rightarrow \mathfrak{B}(\mu)$ follows from the uniformity of the convergence. $\square$

Note that the converse of Lemma 4.1 is also true.

\section{Completion of the proof}

Now suppose that $\mu \in \mathcal{P}(\mathbb{T})$ is IP-Dirichlet along $b$. By Lemma 4.1, $\exists \mathcal{X}: \Omega \rightarrow \mathfrak{B}(\mu)$ satisfying $\left(\$^{\not}\right)$.

We claim that

\section{$(\bullet)$}

$$
\mathcal{X}(\tau \omega)=\chi_{\varphi(\omega)} \mathcal{X}(\omega) .
$$

To see this, note that $\chi_{b(K(\omega) \cap[1, n])}(t)=\prod_{k=1}^{n} \chi_{\omega_{k} b_{k}}(t)=: \mathcal{X}_{n}(\omega, t)$. For $n>\ell(\omega)$,

$$
\frac{\mathcal{X}_{n}(\tau \omega)}{\mathcal{X}_{n}(\omega)}=\frac{\chi_{b(K(\tau \omega) \cap[1, n])}}{\chi_{b(K(\omega) \cap[1, n])}}=\prod_{k=1}^{n} \frac{\chi_{(\tau \omega)_{k} b_{k}}}{\chi_{\omega_{k} b_{k}}}=\chi_{\varphi(\omega)}
$$

Since $\frac{\mathcal{X}_{n}(\tau \omega)}{\mathcal{X}_{n}(\omega)} \underset{n \rightarrow \infty}{\stackrel{\mathfrak{B}(\mu)}{\longrightarrow}} \frac{\mathcal{X}(\tau \omega)}{\mathcal{X}(\omega)}$, this proves $(\dot{\bullet})$.

By $\left(\$^{\infty}\right), \exists n_{J} \rightarrow \infty$ so that

$$
\sum_{J \geq 1}\left\|\mathcal{X}_{n_{J}}-\mathcal{X}\right\|_{L^{2}(P \times \mu)}<\infty \leq \sum_{J \geq 1} \sup _{\omega \in \Omega}\left\|\chi_{b\left(K(\omega) \cap\left[1, n_{J}\right]\right)}-\mathcal{X}(\omega)\right\|_{L^{2}(\mu)}<\infty
$$

(where $\mathcal{X}(\omega, t):=\mathcal{X}(\omega)(t))$. Hence $\mathcal{X}_{n_{J}} \rightarrow \mathcal{X} P \times \mu$-a.e. and by Fubini's theorem, $\exists \Lambda \in \mathcal{B}(\mathbb{T}), \mu(\Lambda)=1$ so that

$$
\prod_{k=1}^{n_{J}} \chi_{\omega_{k} b_{k}}(t) \underset{J \rightarrow \infty}{\longrightarrow} \mathfrak{X}_{t}(\omega)=\mathcal{X}(\omega)(t) \forall t \in \Lambda \& P \text { - a.e. } \omega \in \Omega .
$$

By $(\bullet)$, for $t \in \Lambda$,

$$
\mathfrak{X}_{t} \circ \tau=e^{2 \pi i t \varphi} \mathfrak{X}_{t} \quad P \text { - a.e. }
$$




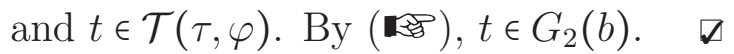

The following example shows that the converse to Theorem 2 is false.

Example 4.2 $\exists b \in \mathbb{N}^{\mathbb{N}}(\uparrow) \& \mu \in \mathcal{P}(\mathbb{T})$ non-atomic, not IP-Dirichlet along $b$ but so that $\mu\left(G_{2}(b)\right)=1$.

Construction:

Define $b \in \mathbb{N}^{\mathbb{N}}(\uparrow)$ by $b_{n}:=\prod_{k=1}^{n} a_{k}$ with $a_{k}:=k+1$.

Consider the mapping $t: \Omega:=\prod_{k \geq 1}\{0,1\} \rightarrow[0,1]$ defined by

$$
t(\omega)=t\left(\omega_{1}, \omega_{2}, \ldots\right):=\sum_{n \geq 1} \frac{\omega_{n}}{b_{n}} .
$$

This is injective and Borel measurable, so $t(\Omega)$ is an uncountable, Borel set in $[0,1]$. We claim that

$$
t(\Omega) \subset G_{2}(b) .
$$

\section{Proof of ( )}

For $\omega \in \Omega$ and $N \geq 1$, we have that

$$
b_{N} t(\omega)=\frac{\omega_{N+1}}{a_{N+1}}+\frac{1}{a_{N+1}} \sum_{k \geq 2} \frac{\omega_{N+k}}{a_{N+2} \ldots a_{N+k}} \quad \bmod 1 .
$$

Now

$$
\left|\sum_{k \geq 2} \frac{\omega_{N+k}}{a_{N+2} \ldots a_{N+k}}\right| \leq \sum_{k \geq 2} \frac{1}{a_{N+2} \ldots a_{N+k}}<\frac{1}{a_{N+1}} .
$$

Thus, we have

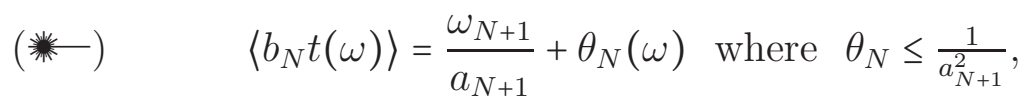

whence $\left|\left\langle b_{N} t(\omega)\right\rangle\right|^{2} \leq \frac{2}{N^{2}}$ and

$$
\sum_{k=1}^{\infty}\left|\left\langle b_{k} t(\omega)\right\rangle\right|^{2} \leq \frac{\pi}{3} \cdot \nabla\left(\frac{\nabla}{}\right)
$$

Now define $P \in \mathcal{P}(\Omega)$ by $P:=\prod_{k \geq 1}\left(\frac{1}{2} \delta_{0}+\frac{1}{2} \delta_{1}\right)$ and set $\mu:=P \circ t^{-1}$, then $\mu \in \mathcal{P}\left(G_{2}(b)\right)$. We now show that $\mu$ is not IP-Dirichlet along $b$.

Fix $1<\lambda<e^{\frac{1}{3}}$, then by (潾-),

$$
\left|\sum_{\lambda^{N}<k<\lambda^{N+1}}\left\langle b_{N} t(\omega)\right\rangle-\sum_{\lambda^{N}<k<\lambda^{N+1}} \frac{\omega_{N+1}}{a_{N+1}}\right| \leq \sum_{\lambda^{N}<k<\lambda^{N+1}} \frac{2}{k^{2}} \underset{N \rightarrow \infty}{\longrightarrow} 0
$$


and setting $s_{n}:=\sum_{j=1}^{n} \omega_{j}, \kappa_{n}:=\left\lceil\lambda^{n}\right\rceil, \ell_{n}:=\left\lfloor\lambda^{n+1}\right\rfloor$, we have

$$
\begin{aligned}
\sum_{\lambda^{N}<k<\lambda^{N+1}} \frac{\omega_{k}}{a_{k}} & =\sum_{\kappa_{N} \leq k \leq \ell_{N}} \frac{s_{k}-s_{k-1}}{k+1} \\
& =\sum_{\kappa_{N} \leq k \leq \ell_{N}} \frac{s_{k}}{k+1}-\sum_{\kappa_{N}-1 \leq k \leq \ell_{N}-1} \frac{s_{k}}{k+2} \\
& =\frac{s_{\ell_{N}}}{\ell_{N}+1}-\frac{s_{\kappa_{N}}}{\kappa_{N}+2}+\sum_{\kappa_{N} \leq k \leq \ell_{N}-1} \frac{s_{k}}{(k+1)(k+2)} .
\end{aligned}
$$

By the SLLN, we have

$$
\frac{s_{N}}{N} \underset{N \rightarrow \infty}{\longrightarrow} \frac{1}{2} \text { a.s. }
$$

Writing $\Gamma_{N} \approx \Delta_{N}$ as $N \rightarrow \infty$ to mean $\Gamma_{N} \approx \Delta_{N} \underset{N \rightarrow \infty}{\longrightarrow} 0$, we have for $P$-a.e. $\omega \in \Omega$, as $N \rightarrow \infty$ :

$$
\begin{aligned}
\sum_{\lambda^{N}<k<\lambda^{N+1}}\left\langle b_{N} t(\omega)\right\rangle & \approx \sum_{\lambda^{N}<k<\lambda^{N+1}} \frac{\omega_{k}}{a_{k}} \\
& =\frac{s_{\ell_{N}}}{\ell_{N}+1}-\frac{s_{\kappa_{N}}}{\kappa_{N}+2}+\sum_{\kappa_{N} \leq k \leq \ell_{N}-1} \frac{s_{k}}{(k+1)(k+2)} \\
& \approx \sum_{\substack{\kappa_{N} \leq k \leq \ell_{N}-1\\
}} \frac{1}{2 k} \\
& \longrightarrow \log \lambda .
\end{aligned}
$$

Thus

$$
\begin{aligned}
\sup _{F \in \mathcal{F}, \min F>\lambda^{N}}\left\|\chi_{b(F)}-1\right\|_{L^{2}(\mu)}^{2} & \geq \mathbb{E}\left(\left|\exp \left[2 \pi i \sum_{\lambda^{N}<k<\lambda^{N+1}} b_{N} t(\omega)\right]-1\right|^{2}\right) \\
& \geq 4 \mathbb{E}\left(\left|\sum_{\lambda^{N}<k<\lambda^{N+1}}\left\langle b_{N} t(\omega)\right\rangle\right|^{2}\right) \\
& \longrightarrow 2 \log \lambda . \quad
\end{aligned}
$$

\section{$\S 5$ REMARKS ON THE THICKNESS OF RIGIDITY SEQUENCES}

Remark 5.1. Rigidity sequences for weakly mixing transformations can be arbitrarily "large" within the limitation of density zero.

It follows from the definitions that for $b \in \mathbb{N}^{\mathbb{N}}(\uparrow)$ a growth sequence,

$$
|\mathrm{FS}(b) \cap[1, n]| \asymp 2^{c(n)}
$$

where $c(n)=\min \left\{k \geq 1: b_{k} \geq n\right\}$ and if $T \in \operatorname{MPT}(X, \mathcal{B}, m)$ is IP-rigid along $b$, one might expect a rigid sequence at least of this thickness. 
Indeed, in this case, by Theorem $2, \sigma_{T}\left(e(\mathfrak{T})^{c}\right)=0$ where $\mathfrak{T}$ is the dyadic tower with growth sequence $b$ (since by $\left.[\mathrm{AN}] e(\mathfrak{T})=G_{2}(b)\right)$. By theorem 4 in [A2] $\exists L \subset \mathbb{N}$ with

$$
T^{n} \underset{n \rightarrow \infty, n \in L}{\stackrel{\mathrm{MPT}}{\longrightarrow}} \operatorname{Id} \& \frac{|L \cap[1, n]|}{2^{c(n)}} \underset{n \rightarrow \infty}{\longrightarrow} \infty
$$

By the Corollary in [A2], $\forall a(n)>0, \frac{a(n)}{n} \underset{n \rightarrow \infty}{\longrightarrow} 0$, there is a weakly mixing $T \in \operatorname{MPT}(X, \mathcal{B}, m)$ and $L \subset \mathbb{N}$ such that

$$
T^{n} \underset{n \rightarrow \infty, n \in L}{\stackrel{\text { MPT }}{\longrightarrow}} \operatorname{Id} \& \frac{|L \cap[1, n]|}{a(n)} \underset{n \rightarrow \infty}{\longrightarrow} \infty .
$$

For more on this phenomenon, see $\S 3$ in [BJLR].

Remark 5.2. Let $b \in \mathbb{N}^{\mathbb{N}}(\uparrow)$ be a growth sequence and suppose that $\mathrm{H}-\operatorname{dim}\left(G_{1}(b)\right)>\alpha$ (where $\alpha \in(0,1)$ and $\mathrm{H}$-dim denotes Hausdorff dimension).

We claim that $\exists T \in$ MPT weakly mixing \& IP-rigid along $b$ with the property that for any sequence $L \subset \mathbb{N}$ along which $T$ is rigid:

$$
\sum_{n=1}^{\infty} \frac{|L \cap[1, n]|}{n^{2-\alpha}}<\infty
$$

Note that it follows from this that $\sum_{n=1}^{\infty} \frac{2^{c(n)}}{n^{2-\alpha}}<\infty$.

\section{Proof of ( $)$}

As in the proof of theorem 1 of [A3], it follows from Frostman's theorem ([Fr], see also $[\mathrm{KS}])$ that $\exists \mu \in \mathcal{P}\left(G_{1}(b)\right)$ so that

$$
\sum_{n=1}^{\infty} \frac{|\widehat{\mu}(n)|}{n^{1-\alpha}}<\infty
$$

Let $T \in \operatorname{MPT}(X, \mathcal{B}, m)$ be the associated Gaussian automorphism. By Proposition 1, $T$ is IP-rigid along $b$.

Suppose that $T$ is rigid along $L \subset \mathbb{N}$. In particular $\exists N \geq 1$ so that $|\widehat{\mu}(n)| \geq \frac{1}{2} \forall n \in L, n>N$. 
It follows that

$$
\begin{aligned}
\sum_{n=1}^{\infty} \frac{|L \cap[1, n]|}{n^{2-\alpha}} & =\sum_{n=1}^{\infty} \sum_{k=1}^{n} 1_{L}(k) \frac{1}{n^{2-\alpha}} \\
& =\sum_{k=1}^{\infty} 1_{L}(k) \sum_{n=k}^{\infty} \frac{1}{n^{2-\alpha}} \\
& \leq \frac{1}{1-\alpha} \sum_{k=1}^{\infty} 1_{L}(k) \frac{1}{k^{1-\alpha}} \\
& \leq \frac{N}{1-\alpha}+\frac{2}{1-\alpha} \sum_{k=N+1}^{\infty} \frac{|\widehat{\mu}(k)|}{k^{1-\alpha}} \\
& <\infty . \quad \nabla(\$)
\end{aligned}
$$

Remark 5.3. The condition ( $)$ is sharp. In $\S 5$ of [A3], $\forall \alpha \in(0,1)$ a growth sequence $b^{(\alpha)} \in \mathbb{N}^{\mathbb{N}}(\uparrow)$ is exhibited with

$$
\mathrm{H}-\operatorname{dim}\left(G_{1}\left(b^{(\alpha)}\right)\right)=\alpha \& 2^{c^{(\alpha)}(n)} \gg n^{1-\alpha}
$$

whence if $T \in \operatorname{MPT}(X, \mathcal{B}, m)$ is so that $\sigma_{T}\left(G_{2}(b)\right)=1$, then (again by theorem 4 in [A2]) $\exists L \subset \mathbb{N}$ rigid for $T$ with $\frac{|L \cap[1, n]|}{n^{1-\alpha}} \rightarrow \infty$ and therefore

$$
\sum_{n=1}^{\infty} \frac{|L \cap[1, n]|}{n^{2-\alpha}}=\infty \text {. }
$$

\section{REFERENCES}

[A1] J. Aaronson, An introduction to infinite ergodic theory; Mathematical Surveys and Monographs, 50. American Mathematical Society Providence, RI(1997)

[A2] J. Aaronson, Rational ergodicity, bounded rational ergodicity and some continuous measures on the circle. A collection of invited papers on ergodic theory. Israel J. Math. 33 (1979), no. 3-4, 181-197 (1980).

[A3] J. Aaronson, The eigenvalues of non-singular transformations. Israel Journal of Math. 45, 297-312 (1983).

[AN] J. Aaronson, M.G. Nadkarni, $L_{\infty}$ eigenvalues and $L_{2}$ spectra of non-singular transformations, Proc. London Math. Soc. 93055 91987), 538-570.

[BJLR] V. Bergelson, A. del Junco, M. Lemańczyk, J. Rosenblatt, Rigidity and non-recurrence along sequences, submitted (arxiv.org/pdf/1103.0905).

[CFS] I.P. Cornfeld, S.V. Fomin, Y.G. Sinai, Ergodic Theory, Springer-Verlag, New York, 1982.

[E] H. G. Eggleston, Sets of fractional dimensions which occur in some problems of number theory, Proceedings of the London Mathematics Soc (2) 54 (1952) 42-93.

[EG] T. Eisner, S. Grivaux,Hilbertian Jamison sequences and rigid dynamical systems, J. Functional Analysis 261 (2011), 2013-2052.

[ET] P. Erdős and S. J. Taylor, On the set of points of convergence of a lacunary trigonometric series and the equidistribution properties of related sequences, Proceedings of the London Mathematics Society (3) 7 (1957) 598-615. 
[Fr] O. Frostman, Potentiel d'equilibre et capacite des ensembles, Thesis, Lund, 1935

[Fu] H. Furstenberg, Recurrence in Ergodic Theory and Combinatorial Number Theory, Princeton University Press, Princeton, New Jersey, 1981.

[FW] H. Furstenberg, B. Weiss, The finite multipliers of infinite ergodic transformations, in "The structure of attractors in dynamical systems" (Proc. Conf., North Dakota State Univ., Fargo, N.D., 1977), pp. 127 -132, Lecture Notes in Math. 668, Springer, Berlin, 1978.

[HS] N. Hindman, D. Strauss, Algebra in the Stone-C̆ech compactification, De Gruyter, 1998

[HMP1] B. Host, J.-F. Mela and F. Parreau. Analyse harmonique des mesures. Asterisque 135-136, SMF, (1986).

[HMP2] B. Host, J.-F. Mela and F. Parreau. Nonsingular transformations and spectral analysis of measures. Bull. Soc. Math. France 119 (1991), no. 1, 33-90.

[IN] Ito, Sh. ; Nakada, H. Approximations of real numbers by the sequence $\{n \alpha\}$ and their metrical theory. Acta Math. Hungar. 52 (1988), no. 1-2, 91-100.

[KS] J. P. Kahane and R. Salem, Ensembles parfaites et series trigonometriques, Hermann, Paris, 1963.

$[\mathrm{KT}]$ A. Katok and J.-P. Thouvenot, Spectral properties and combinatorial constructions in ergodic theory, Handbook of Dynamical Systems. Vol. 1B, 649743, Elsevier, Amsterdam, 2006.

[N1] Nadkarni, M. G. Basic ergodic theory. Hindustan Book Agency, New Delhi, 1998.

[N2] M.G. Nadkarni, Spectral Theory of Dynamical Systems, Hindustan Book Agency, New Delhi, 1998.

[P] F. Parreau, Ergodicité et pureté des produits de Riesz, Ann. Inst. Fourier, 40 (1990) 391-405.

[Z] R.J. Zimmer, Ergodic theory and semisimple groups, Monographs in Mathematics, vol 81, Birkhäuser Verlag, Basel, 1984.

(Aaronson) School of Math. Sciences, Tel Aviv University, 69978 Tel Aviv, Israel.

E-mail address: aaro@tau.ac.il

(Hosseini) Faculty of Mathematical Science, University of Guilan, RASHT, IRAN

E-mail address: hoseini@guilan.ac.ir

(Lemańczyk) Faculty of Mathematics and Computer Science, Nicolaus Copernicus University, ul. Chopina 12/18, 87-100 Toruń, Poland

E-mail address: mlem@mat.uni.torun.pl 